\title{
SIGNIFICADOS E PRÁTICAS DIETÉTICAS ENTRE IDOSOS DIAGNOSTICADOS COM DIABETES MELLITUS TIPO 2
}

\section{Meanings and dietary practices among older adults diagnosed with type 2 diabetes mellitus}

\section{Significados y prácticas de dietas entre mayores con diagnóstico de diabetes mellitus del tipo 2}

Nathielle Brasileiro de Souza Rocha (iD

Universidade Estadual do Sudoeste da Bahia - UESB - Jequié (BA) - Brasil

Andréa dos Santos Souza iD

Universidade Estadual do Sudoeste da Bahia - UESB - Jequié (BA) - Brasil

Caliana Beatriz de Aguiar Barbosa (iD

Universidade Estadual do Sudoeste da Bahia - UESB - Jequié (BA) - Brasil

Edmeia Campos Meira iD

Universidade Estadual do Sudoeste da Bahia - UESB - Jequié (BA) - Brasil

Juliana da Silva Oliveira (iD

Universidade Estadual do Sudoeste da Bahia - UESB - Jequié (BA) - Brasil

Isleide Santana Cardoso Santos (iD

Universidade Estadual do Sudoeste da Bahia - UESB - Jequié (BA) - Brasil

Laiza Carvalho Costa iD

Universidade Estadual do Sudoeste da Bahia - UESB - Jequié (BA) - Brasil

\section{RESUMO}

Objetivo: Compreender os significados e as práticas dietéticas entre pessoas idosas com diabetes mellitus tipo 2 (DM2). Métodos: Pesquisa qualitativa, realizada entre os meses de setembro de 2016 e março de 2017, por meio de entrevista semiestruturada com 20 idosos diagnosticados com DM2, residentes na cidade de Jequié, Bahia, Brasil. As informações foram tratadas pelo método de análise de conteúdo, modalidade temática, na qual emergiram as seguintes categorias: Decisões dos idosos com DM2 frente às recomendações dietéticas; Significados atribuídos à dieta prescrita por idosos com DM2; "Saio da linha": flexibilidade nas práticas dietéticas; Facilidades e dificuldades no gerenciamento da dieta. Resultados: Apesar dos idosos compreenderem a importância da dieta, diversas são as formas como agem diante das recomendações dietéticas, desde a adesão total até a não adesão. A dieta é percebida como de difícil tolerância, sem sabor, controlada, repugnante, desvinculada das preferências, capaz de causar sentimentos como tristeza, sensação de fraqueza e morte. Os idosos flexibilizam as recomendações dietéticas, buscando adaptar-se a sua condição de saúde. O manejo da dieta demonstrou ser central e complexo, haja vista a necessidade de uma ruptura cultural. Conclusão: Os idosos reinterpretam e adaptam as explicações biomédicas, pois valorizam a cultura, a sociabilidade e o prazer sensorial. Práticas intersetoriais, interdisciplinares e inovadoras são recomendadas para resultados mais efetivos no gerenciamento da dieta.

Descritores: Diabetes Mellitus; Doença Crônica; Alimentos, Dieta e Nutrição; Conhecimentos, Atitudes e Práticas em Saúde.

\section{ABSTRACT}

Objective: To understand the meanings and dietary practices among older adults with type 2 diabetes mellitus (T2D). Methods: This qualitative study was conducted between September 2016 and March 2017 using through semi-structured interview with 20 older adults diagnosed with Type 2 Diabetes Mellitus (T2D) living in the city of Jequié, Bahia, Brazil. The information was submitted to thematic content analysis and the following categories emerged: T2D older adults' decisions regarding dietary requirements; Meanings given by T2D older adults to prescribed diets; "I get out of line": flexibility in dietary practices; Facilities and difficulties

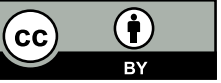


in diet management. Results: Although the older adults understand the importance of diet, they behave in several different ways in relation to dietary requirements, ranging from full adherence to non-adherence. Diet is perceived as difficult, tasteless, controlled, disgusting and does not consider their preferences, thus causing feelings such as sadness, feelings of weakness and death. The older adults are flexible towards dietary requirements and seek to adapt to their health condition. Diet management was shown to be centralized and complex given the need for a cultural break. Conclusion: The older adults reinterpret and adapt the biomedical explanations as they value culture, sociability and sensory pleasure. Intersectoral, interdisciplinary and innovative practices are recommended for more effective diet management outcomes.

Descriptors: Diabetes Mellitus; Chronic Disease; Diet, Food, and Nutrition; Health Knowledge, Attitudes, Practice.

\section{RESUMEN}

Objetivo: Comprender los significados y las prácticas de dieta entre mayores con diabetes mellitus tipo 2 (DM2). Métodos: Investigación cualitativa realizada entre los meses de septiembre de 2016 y marzo de 2017 a través de entrevista semiestructurada con 20 mayores con el diagnostico de DM2, de la ciudad de Jequié, Bahia, Brasil. Se ha tratado las informaciones a través del análisis de contenido en la modalidad temática de la cual emergieron la categorías a continuación: Decisiones de los mayores con DM2 delante las recomendaciones de dieta; Significados de la dieta delante las recomendaciones de dieta; Significados de la dieta para los mayores con DM2; "Salgo de la línea": flexibilidad en las prácticas de dieta; Facilidades y dificultades para la gestión de la dieta. Resultados: Aunque los mayores comprendan la importância de la dieta, son muchas las formas cómo reaccionan delante las recomendaciones de dieta desde la adhesión total hasta la no adhesión. Se percibe la dieta como de tolerancia difícil, sin sabor, controlada, repugnante, desvinculada de las preferencias, capaz de causar sentimientos como la tristeza, la sensación de flaqueza y de muerte. Los mayores flexibilizan las recomendaciones de dietas buscando adaptarse a su condición de salud. El manejo de la dieta ha demostrado ser central y complejo una vez que hay la necesidad de una ruptura cultural. Conclusión: Los mayores reinterpretan y adaptan las explicaciones biomédicas pues valoran la cultura, la sociabilidad y el placer sensorial. Las prácticas intersectoriales, interdisciplinares e innovadores son recomendadas para resultados más efectivos en la gerencia de la dieta.

Descriptores: Diabetes Mellitus; Doença Crônica; Alimentos, Dieta e Nutrição; Conhecimentos, Atitudes e Práticas em Saúde.

\section{INTRODUÇÃO}

O diabetes mellitus tipo 2 (DM2) tem se destacado como um problema de saúde pública de elevado custo para o paciente, a família e a sociedade, e também para o Sistema Único de Saúde (SUS). Esse fenômeno é decorrente do envelhecimento populacional, da maior urbanização, da elevada prevalência de obesidade e sedentarismo, bem como da maior sobrevida das pessoas com DM2(1).

A prevalência de diabetes aumenta com o avanço da idade. Segundo a Federação Internacional de DM, existe 326,5 milhões de pessoas em idade laboral (20 a 64 anos) e 122,8 milhões de pessoas de 65 a 99 anos com DM(2). O DM, classifica-se em tipo 1 (DM1), tipo 2 (DM2), gestacional e outros formas de apresentação. Entretanto, a forma mais comum, verificada em 90-95\% dos casos, é o DM2, caracterizado por defeitos na ação e secreção da insulina (1).

O diabetes está incluído entre as doenças classificadas como crônicas não transmissíveis (DCNT), caracterizadas por serem multifatoriais, se desenvolverem no decorrer da vida e serem de longa duração. Dessa forma, pela impossibilidade de cura, as DCNT são passíveis de controle por meio da aquisição de hábitos saudáveis, uso de medicamentos e reformulação do cotidiano, adaptando rotinas para que obtenha controle da enfermidade e qualidade de vida, prevenindo ou postergando complicações. Entre essas formas de controle relacionadas aos hábitos de vida, destaca-se a alimentação, que está presente no gerenciamento do diabetes como elemento central tanto para a compreensão de sua causa como para explicar o grau de controle sobre a enfermidade ${ }^{(3)}$.

Considerando a relevância da alimentação e o impacto causado no cotidiano das pessoas com diabetes mellitus tipo 2, a dieta foi analisada com ênfase nas dificuldades para a mudança, na pressão moral a que são submetidos os que estão em tratamento( ${ }^{(4)}$ e na subjetividade encontrada nos modelos explicativos ${ }^{(5)}$. Pessoas que convivem com diabetes DM2 percebem que o comportamento alimentar é uma barreira que deve ser superada para obter melhor condição de saúde e sucesso no tratamento ${ }^{(5)}$.

Deve-se, ainda, considerar que a hipertensão arterial sistêmica e o diabetes mellitus se destacam por serem grandes problemas de saúde pública, estando entre os cinco principais riscos globais para a mortalidade no mundo(6). Ademais, de acordo com a Política Nacional de Promoção da Saúde (PNPS), deve-se considerar a autonomia e singularidade dos pacientes, pois a forma como eles elegem seus modos de viver depende não apenas da vontade, ou liberdade, individual e comunitária, pois estão condicionados e determinados pelos contextos social, econômico, político e cultural em que eles vivem ${ }^{(7)}$. 
Este estudo justifica-se pelo impacto do controle do DM2 está nas recomendações nutricionais e os pacientes idosos com DM2 podem alimentar-se erroneamente. Além disso, podem desconhecer sobre o tema. Assim, a abordagem do manejo nutricional do paciente com DM2 não deve ser somente voltado à dieta, mas ter um estilo subjetivo, de olhar comportamental, colocando o paciente no centro do cuidado.

Portanto, com o intuito de investigar essa problemática, questiona-se: quais os significados e as práticas dietéticas relacionadas ao paciente idoso com DM2? Dessa forma, esta pesquisa tem por objetivo compreender os significados e as práticas dietéticas de pessoas idosas com diabetes mellitus tipo 2.

\section{MÉTODOS}

Trata-se de uma pesquisa descritiva e qualitativa ${ }^{(8)}$ derivada de um projeto de pesquisa matricial denominado Significados e práticas de cuidados em saúde entre pessoas idosas com diabetes (SIPRACID), vigente no período de março de 2016 a dezembro de 2018, realizado por docentes e estudantes de graduação da Universidade Estadual do Sudoeste da Bahia, Brasil.

Os participantes do estudo foram 20 idosos com diagnóstico médico de DM2, de ambos os sexos, atendidos no Sistema de cadastramento e acompanhamento de hipertensos e diabéticos - Hiperdia. Adotaram-se como critérios de inclusão: ter idade igual ou superior a sessenta anos, possuir diagnóstico médico de DM2 estabelecido por um período mínimo de um ano e estar cadastrado no Hiperdia. O referido programa destina-se a cadastrar e acompanhar pessoas que têm diagnóstico de hipertensão arterial e/ou diabetes mellitus atendidos na rede ambulatorial do Sistema Único de Saúde - SUS, o qual permite produzir informações epidemiológicas visando à aquisição, dispensação e distribuição de medicamentos aos pacientes cadastrados em uma determinada área adscrita, bem como a redução da morbimortalidade desses agravos à saúde ${ }^{(9)}$.

Utilizou-se como critério de exclusão a apresentação de déficit cognitivo na avaliação do miniexame do estado mental (MEEM). Esse instrumento deriva-se da necessidade de uma avaliação padronizada, simplificada, reduzida e rápida no contexto clínico do estado mental, especificamente dos sintomas referentes à demência ${ }^{(10)}$.

O centro de saúde eleito para seleção dos participantes fica localizado na área urbana do município de Jequié, Bahia, Brasil, e se constitui em lócus para as práticas de campo de universidades, faculdades e cursos para formação técnica em saúde. Além disso, possui implantado o atendimento às pessoas com DM há quase vinte anos, atendendo a 459 pessoas cadastradas no Hiperdia. Os atendimentos às pessoas com DM funcionam nos turnos matutino e vespertino, com a participação de enfermeiras, médicos clínicos, nutricionistas e agentes comunitários de saúde. As ações desenvolvidas são, predominantemente, de enfoque biomédico, individual ou com participação do familiar, e seguem orientações contidas no protocolo do Ministério da Saúde ${ }^{(11)}$.

Em sua maioria, o encontro com os participantes se deu a partir de convite pessoal e individual na sala de espera do Hiperdia. No entanto outros métodos de acesso foram utilizados, de modo complementar, tais como: visitas domiciliares a pessoas com diabetes mellitus tipo2, localizadas a partir do registro de cadastramento no serviço; indicação por parte dos próprios idosos participantes; e por intermédio de agente comunitário de saúde, que disponibilizou dados de sua microárea atualizados com o nome de idosos com DM2 e seus respectivos endereços.

A coleta de informações foi realizada no período de setembro de 2016 até março de 2017, por meio de entrevistas semiestruturadas $^{(8)}$, nas quais utilizou-se um instrumento com perguntas que questionavam aspectos relacionados à dieta no gerenciamento do DM2 entre idosos, como: licenças sociais; quem compra e prepara os alimentos; e quais as preferências, as rejeições, os significados, as resistências, as dificuldades e as facilidades para o gerenciamento da alimentação.

Para definição do encerramento das entrevistas, utilizou-se o critério de saturação de dados, quando houve a percepção de que as falas se tornavam constantes sobre o objeto questionado ${ }^{(12)}$. As entrevistas variaram de 30 minutos a $1 \mathrm{~h} 20$ minutos, todas concedidas no domicílio, em dias e horários acordados entre os participantes. Os registros foram realizados por meio de um gravador de voz digital e a transcrição respeitou as falas na íntegra, realizando apenas algumas correções para a obtenção de uma leitura mais fluida.

Para a interpretação dos dados, seguiram-se as etapas da Análise Temática de Conteúdo ${ }^{(13)}$. Houve sucessivas leituras flutuantes das informações, seguidas de registro das observações dos elementos estruturantes e convergentes, das temáticas afins e das contradições. Em seguida, agruparam-se em arquivos diferentes as temáticas afins, facilitando a identificação em subconjuntos de dados dos elementos que retrataram a interpretação dos idosos sobre o tema em questão(13). 
Após a análise das entrevistas, emergiram quatro categorias temáticas: Decisões dos idosos com DM2 frente às recomendações dietéticas; Significados atribuídos à dieta prescrita por idosos com DM2; "Saio da linha": flexibilidade nas práticas dietéticas, e Facilidades e dificuldades no gerenciamento da dieta.

A fim de preservar a identidade dos entrevistados, foi adotada a letra "E" (entrevistados) seguida por algarismo numérico de acordo com a sequência cronológica da realização da entrevista. A pesquisa obteve a aprovação do Comitê de Ética em Pesquisa da Universidade Estadual do Sudoeste da Bahia, Parecer n. ${ }^{\circ}$ : 1.535.559, e os idosos assinaram o Termo de Consentimento Livre e Esclarecido. Aqueles que não eram alfabetizados colocaram a impressão dactiloscópica.

\section{RESULTADOS E DISCUSSÃO}

A partir da análise das entrevistas e divisão dos núcleos de sentido, emergiram as seguintes categorias:

\section{Decisões dos idosos com DM2 frente às recomendações dietéticas}

Esta categoria, decisões dos idosos com DM2 frente às recomendações dietéticas, traz a informação que, após confirmação e comunicação do diagnóstico de DM2, a pessoa é orientada a adotar um novo estilo de vida, no qual a alimentação tem grande relevância e impacto no seu controle da doença. A prescrição dietética é realizada pela nutricionista e recomendada também na consulta com os demais membros da equipe de saúde. No entanto, os idosos demonstraram modos distintos de reagir às orientações, variando da disposição total, ou parcial, até a completa indisposição para adequar a alimentação, conforme é possível notar nos seguintes depoimentos:

"Não como açúcar, tomo adoçante. Não como gordura, nem fritura." (E16)

"Sigo a dieta. De vez em quando como um pedaço de bolo, mas não é todo dia, é de vez em quando." (E13)

"Não me privo de nada. Não considero uma obrigação seguir a dieta para diabéticos. Não é difícil a alimentação não, porque eu como macarrão, como tudo: arroz, feijão... e o feijão é com carne dentro." (E8)

Os resultados demonstram que mesmo as pessoas dispostas, inicialmente, a mudar seu estilo alimentar, essa disposição para mudança se altera no curso da doença, revelando ser intensa no período inicial, quando é recebido o diagnóstico de DM2, mas com uma tendência a ser menos rigorosa com o passar do tempo, como afirmaram os participantes:

"Não sigo rigorosamente. No início segui muito, mas depois comecei a falhar um pouco. Às vezes como uma coisinha a mais." (E5)

As mudanças relatadas na alimentação foram associadas à quantidade, à qualidade e ao preparo dos alimentos:

"Minha comida é cozida ou grelhada. Eu como limitado. Meu arroz não é integral, mas ele não tem nada. Não tem sal, nem nada. O feijão, se colocar carne e gordura, eu não como." (E17)

Frente às recomendações dietéticas necessárias ao controle da doença, é exigido do paciente uma tomada de decisão, demonstrando haver por parte dele uma grande responsabilidade no tratamento. No entanto, nem todos apresentam a imediata disposição para mudar o comportamento e, quando essa atitude não é compreendida, aumentam as chances de frustração, impaciência por parte do profissional e de insucesso na adoção de novo estilo de vida por parte do adoecido. A pessoa com DM2 precisa desejar a mudança e almejar mantê-la. No entanto, é preciso respeitar sua autonomia, estimulando-a a encontrar soluções viáveis para os problemas que a impedem de alcançar as metas negociadas.

Os participantes do atual estudo afirmaram conviver com o DM2 por tempo igual ou superior a dezesseis anos. Portanto, já passada a fase inicial e situados em uma vivência prolongada com a doença, cuja dinâmica da vida evoca decisões diárias relacionadas às orientações dietéticas.

Também foram encontrados resultados semelhantes aos de pesquisa realizada com 45 pacientes com DM2 no México, em que se observaram três estágios na mudança dos hábitos alimentares: inicial, acomodação-adaptação e crise. No estudo mexicano, as autoras identificaram que os primeiros conflitos dos pacientes com DM2 relacionados à mudança de hábitos alimentares são vivenciados quando ocorre a confirmação do diagnóstico e uma dieta é prescrita, momento em que podem adotar diferentes padrões: descrença e rejeição ao tratamento; aceitação, com mudança imediata da dieta, e posterior abandono após um curto período de tempo, ou o paciente abandona a dieta depois de um longo período de tempo ${ }^{(14)}$. 
A educação e o estilo de vida saudável (dieta e atividade física) fazem parte da conduta terapêutica para todos os pacientes com diabetes. Em alguns casos, pode ser obtido controle glicêmico adequado exclusivamente com as mudanças no estilo de vida, sem a necessidade diária de comprimidos ou insulina ${ }^{(1)}$.

Essa evidência científica reforça a relevância dos profissionais de saúde em apoiarem os pacientes no processo de mudança nos hábitos de vida, especialmente no estágio inicial da doença ou na confirmação do diagnóstico.

\section{Significados atribuídos à dieta prescrita por idosos com DM2}

Esta categoria, significados atribuídos à dieta prescrita por idosos com DM2, revela que os participantes reconhecem a centralidade e a relevância da dieta no controle do DM2:

"Mas eu acho assim, que o diabetes, se eu aguentasse seguindo a dieta direitinho, eu já tinha sarado." (E20)

Em parte, esse reconhecimento se deve à associação do DM2 a um comportamento alimentar inadequado, caracterizado pelo consumo excessivo, especialmente de carboidrato (açúcar), como se pode notar no seguinte discurso:

"Mas eu perguntei: 'Doutora, eu fiquei com diabetes por causa de doce?'Aí ela disse que não, porque o corpo tem a capacidade de extinguir o açúcar. Só não extingue quando a pessoa é diabética." (E2)

Desse modo, é compreensível que, para alguns dos participantes, a restrição principal necessária para o controle do DM2 é a de açúcar/doce:

"Como batata, banana, inhame, aipim... Eu gosto dessas coisas e como. Mas meu café faço amargo para tomar com adoçante." (E1)

A dieta restrita e adequada para o controle do DM, sob a ótica dos idosos participantes deste estudo, significa algo pesaroso, difícil de tolerar, sem sabor, desesperador, repugnante, desvinculada das preferências, capaz de causar tristeza, sensação de fraqueza e morte:

"A dieta é tão diferente que você não aguenta. Você se sente fraca, você sente fome, aí ela vence a gente. [...] A panela está lá, feita de arroz integral, com o quê dentro? Com jiló! Purgante, né?" Enquanto os outros estão com uma panela de frango, uma bandeja de frango assado, e você vê o jiló e ter que comer aquilo ali com feijão de corda [...]. 'É, doutor, agora eu vou morrer, não vou poder comer mais nada?'A gente pensa que se parar de comer, morre." (E20)

"A comida perde o sabor." (E18)

Ficou demonstrada a centralidade da dieta na vida do grupo de idosos entrevistados ao associarem uma alimentação inadequada à causa e ao descontrole do DM2. Os doces foram falados como irresistíveis em virtude do hábito de consumi-los desde a infância.

Na perspectiva biomédica, o consumo de doces em excesso é considerado um marcador de padrão de alimentação não saudável. O açúcar é um alimento extremamente calórico e, se consumido em grandes quantidades, pode favorecer a presença de sobrepeso e obesidade, importantes fatores de risco para o diabetes ${ }^{(15)}$. No entanto, entre as pessoas idosas com diabetes entrevistadas neste estudo, os doces foram reportados como alimentos apreciados que faziam parte dos seus hábitos alimentares em sua trajetória de vida, simbolizando o prazer, a satisfação de um desejo e alegria, constituindo um conflito abolir ou reprimir seu consumo.

Os significados negativos atribuídos à dieta recomendada para as pessoas idosas com DM2 também foram reportados em outros estudos envolvendo a participação de pessoas adultas, o que demonstra ser um significado compartilhado pelos que convivem com a doença, independentemente da idade ou sexo ${ }^{(14,16)}$.

Esses significados devem ser analisados considerando o valor social atribuído à liberdade, como parte da essência humana. Ser livre para comer o que se quer, quando e quanto se quer é um aspecto muito valorizado, pois a comida para os idosos é um elemento-chave para o gozo e bem-estar na velhice, por estar impregnada de afeto, emoção, alegria e sociabilidade ${ }^{(17)}$.

A restrição da liberdade e do prazer contribui, portanto, para a construção dos significados negativos ("difícil de tolerar", "sem sabor", "repugnante", "desvinculada das preferências", "tristeza", "sensação de fraqueza" e "morte"), sendo um conflito constante. De modo que, em alguns momentos, o adoecido vence e, em outros, é vencido pelo gosto e prazer, pois é muito complexo abrir mão de alimentos que tinha prazer em comer ${ }^{(18)}$. 
Para os idosos analisados, as restrições alimentares necessárias ao controle de doenças crônicas, como o DM2 e a hipertensão arterial, podem ser encaradas como mais uma entre as perdas na velhice, encaradas como "pequenas mortes" impostas pelo adoecimento. No presente estudo, a morte associada à dieta tem uma conotação real para os participantes que acreditam ser decorrente da fraqueza e escassez de nutrientes necessárias ao corpo biológico quando se adota uma restrição rigorosa. Todavia aparece também na trajetória de adoecimento crônico, como elemento figurado, capaz de ressignificar a existência a partir das experiências que remetem a atributos da morte concreta, como ruptura, interrupção e tristeza ${ }^{(19)}$.

No estudo em questão, percebeu-se que o termo dieta associado pelos entrevistados à conotação negativa de restrição, alimento insípido, controle, desprazer, reservada a pessoas doentes ou com risco de adoecer, ou seja, possuidoras de uma condição que as torna diferente dos outros. Desse modo, parece estar construído socialmente que dieta é ruim, dissociada do prazer, vinculada ao valor nutricional necessário ao corpo biológico, mas, muitas vezes, isolada do corpo social.

\section{"Saio da linha": flexibilidade nas práticas dietéticas}

Esta categoria, "Saio da linha": flexibilidade nas práticas dietéticas, permite afirmar que existe uma nítida diferença entre a dieta prescrita pela nutricionista (também orientada pelos demais profissionais de saúde) e a dieta adotada no cotidiano. Embora o profissional seja reconhecido por seu saber e competência, a escolha de seguir, ou não, a dieta, compete (em parte) ao adoecido. Assim, a legitimidade do saber biomédico não garante "obediência" estrita. A dieta como é pensada e vivida pelos idosos participantes demonstra inúmeras nuances de interpretação: comer limitado, não abusar, não exagerar, beliscar, petiscar, comer um pouquinho, não extravasar, experimentar:

"Se tiver uma festa de aniversário, eu vou e como (risos). Agora, se o bolo estiver muito doce, eu experimento, mas não como." (E14)

Portanto, quando questionados se estão seguindo a dieta, respondem positivamente com base em seu modelo explicativo sobre o tratamento e no modo como a adaptam à sua realidade:

"Sigo a dieta e, de vez em quando, como um pedaço de bolo, mas não é todo dia, é de vez em quando." (E13)

Sobre a flexibilidade e adaptação das prescrições dietéticas, afirmam cumpri-las quase sempre, porque consideram que não é ameaçador comer de vez em quando, "uma vez ou outra". Confessam: "dou uma 'puladinha', não é 100\%, não sigo à risca, como uma coisinha a mais, saio da linha, como só um pedacinho", conforme se vê nos relatos:

"Mas abusar, eu não abuso, não. Agora, de vez em quando, dou uma puladinha, assim, em um negócio diferente (risos) [...] Minha nora faz uma feijoada, eu como, sabe por quê? Uma vez por semana não faz mal, não!" (E6)

No gerenciamento da dieta é possível notar com maior frequência um cumprimento parcial das prescrições do que rejeição total delas. Àqueles que cometem "deslizes", se autodenominam "traquinas", "relaxados". Assumem que cometem "pecados" no que tange à alimentação que resultam em descontrole glicêmico:

"É porque eu sou 'traquina'. Como coisa que não deve." (E7)

"Eu também dei uma relaxada, comi de tudo e, por isso, subiu mais um pouquinho. Porque eu estava lá na roça, comendo banana da terra, jaca dura (risos), aí ela (glicemia) subiu, foi pra 180." (E3)

No entanto, a consciência da transgressão nem sempre conduz a uma mudança de comportamento, porque, para muitos, é tolerável e compreensível que não resistam continuamente a uma prescrição tão rigorosa:

"Ela (diabetes) dá uma caída boa, volta até o normal. Mas a gente, que tem diabetes, aproveita que está baixo e volta a comer pensando que já sarou." (E20).

E, para fugir do julgamento e das cobranças dos profissionais, adotam como estratégia faltar às consultas e não dizer a verdade:

"Agora, em fevereiro, eu vou pra consulta. Dezembro e janeiro eu não fui, porque eu sei que eu fiz errado, comi demais coisa que não podia; fui pra roça, eu comi jaca na roça." (E20)

Dos idosos com DM2 investigados, aqueles que não atribuíram, ou manifestaram menor dificuldade, justificaram a falta de apetite. Eles relataram, quase que invariavelmente, ser o seguimento da dieta uma tarefa muito difícil. 
A manifestação de crises, ou complicações ameaçadoras da vida, que lhes impõe a adesão a fim de garantir a sobrevivência.

Foram múltiplos e coexistentes os argumentos apresentados pelos idosos do atual estudo para transgredir as recomendações dietéticas. No entanto pode-se notar em suas falas certo grau de conhecimento sobre alimentos de alto e baixo índice glicêmico ao defini-los e polarizá-los como certo e errado, o que deve e o que não deve, o que pode e o que não pode. Sendo assim, o problema parece estar situado além do campo exclusivo da informação, como também ficou demonstrado em estudo realizado na região Nordeste do $\operatorname{Brasi}^{(20)}$.

Ainda no contexto brasileiro, pesquisadores identificaram que, em cidade do Rio Grande do Sul, a motivação para adoção de hábitos saudáveis relacionados à alimentação entre pessoas com doenças crônicas se constitui em um desafio para profissionais de saúde, em virtude da associação da dieta com elementos complexos, como tradição familiar de alimentação, sensação de desconforto no ambiente de convivência social e facilidade de acesso a alimentos calóricos industrializados ${ }^{(21)}$.

Observou-se, no atual estudo, uma rejeição aos alimentos integrais, o que se deve ao fato de que esses não foram habituais durante a vida do grupo investigado, favorecendo a percepção de sabor ruim e incomum ao seu paladar. Resultados similares foram encontrados em um estudo realizado no Ceará, que notou um baixo consumo de alimentos integrais ${ }^{(22)}$. Outros fatores, contudo, podem favorecer a recusa aos integrais, como crenças sobre esses alimentos, questões econômicas e a falta de experiência com o modo de preparo. De um lado estava o alimento integral, ideal, natural, menos processado, mais saudável e rico em nutrientes. Em oposição, o alimento industrializado, mais processado, com menor teor de fibra, maior índice glicêmico. Essa dicotomia, cuja escolha é vivenciada como conflito, foi percebida nas falas dos investigados ao se referirem ao macarrão, arroz e pão integrais ou macarrão, arroz branco e pão francês. Alimentos mais saudáveis, ricos em fibras e pobres em açúcares, são mais $\operatorname{caros}^{(20)} \mathrm{e}$, na realidade de pessoas de baixa renda, como as que participaram da atual pesquisa, existe dificuldade para incluí-los diariamente nas suas refeições.

Os eventos sociais, como os aniversários, casamentos, reuniões de família nos finais de semana, em datas comemorativas, encontro de amigos ou eventos da igreja, foram desafiadores para os idosos. Nessas circunstâncias, são servidos, em abundância, alimentos excluídos da prescrição dietética, mas de grande valor simbólico, como é o caso do bolo da noiva, dos doces e da feijoada com a família. A prática permissiva de "licenças sociais" remete à associação entre a alimentação e a sociabilidade entre os idosos com DM2. Nessa perspectiva, para além dos aspectos nutricionais e biológicos, a alimentação e a comensalidade evocam o convívio familiar, o estreitamento de laços sociais, a afetividade e uma forma de hospitalidade ${ }^{(23)}$.

\section{Facilidades e dificuldades no gerenciamento da dieta}

Nesta categoria, facilidades e dificuldades no gerenciamento da dieta, os participantes deste estudo demonstram que, para o gerenciamento da dieta, torna-se mais fácil quando apresentam o apetite reduzido:

"Também, agora não estou comendo quase nada. Até aquele arroz branco não quero nem ver! Quando eu tinha apetite, eu comia dois pães com manteiga e, agora, não estou comendo nenhum!" (E7).

Em relação a dificuldades em seguir a prescrição dietética, elencaram múltiplos fatores de natureza psicossocial, cultural, econômica e pessoal, tais como: participação em eventos sociais, morar só, gênero, resistir às preferências, ansiedade, crenças negativas que associam dieta à morte, gostar de doces, preparar uma refeição diferente para os demais membros da família, escassez de recursos, rejeição pelos alimentos integrais e ruptura cultural alimentar. Tais dificuldades para o gerenciamento da dieta foram assim apresentadas pelo grupo de idosos pesquisado:

"Ah, quando tem um aniversário, eu como um pedacinho de bolo. Não muito, sabe, mas não deixo de comer." (E5) "Quando eu recebo meu benefício, eu passo no mercado, pago e compro mais. Aí pronto, só posso comprar mais no outro mês." (E3)

"É porque a minha natureza para comer essas coisas integrais é ruim. Não gosto." (E4)

"Tem um paciente que morreu de fome, não comia de tudo com medo, e morreu. Já estava seco fazendo regime." (E8)

"As coisas que eu erro só são as que eu te disse: como a cocada de cacau e o doce de leite." (E20)

Das pessoas entrevistadas, as mulheres afirmavam que elas mesmas preparavam sua alimentação: 
"Minha comida sou eu quem faço. Às vezes, de manhã cedo, eu vou pro fogão, e só saio às $10 h$ da beira do fogão." (E12)

Os homens entrevistados não cozinhavam, pois essa tarefa era desempenhada sempre por alguém do sexo feminino (esposa, irmã ou sobrinha) e, segundo eles, elas adequam a refeição conforme prescrito: hipocalórica, hipossódica e hipolipídica.

No presente estudo, constatou-se que a normalidade foi alcançada adaptando a dieta e permitindo ingerir em quantidades menores os alimentos servidos. As dificuldades encontradas em manter a prática do cuidado de si nas situações de reunião familiar foram também reportadas entre idosos.

Morar sozinho, não ter a presença de um familiar corresidindo, foi apontado como uma dificuldade para resistir a alimentos restritos, como os doces. A presença de um familiar parece reforçar o papel da heterovigilância, motivando os idosos com DM2 a seguirem a dieta prescrita, além de que a presença de outro pode motivar o alimentar-se por ter com quem compartilhar as refeições. Pessoas significativas tendem a exercer uma influência positiva de cooperação e incentivo, embora poucos também possam desempenhar papéis neutros ou inibidores. Em outros contextos e grupos etários, os homens contribuíram com as mudanças de comportamentos nutricionais de mulheres. Isto ficou demonstrado na investigação cujas participantes foram mulheres com DM2, maiores de 18 anos, de origem latina, residentes em Los Angeles, Califórnia, Las Vegas e Nevada, que afirmaram que os seus maridos tiveram a maior influência em suas atitudes e escolhas ${ }^{(24)}$.

Outro aspecto apresentado no presente estudo foi que preparar e servir refeições diferenciadas para os outros membros da família se constituíram em situações tentadoras para um comportamento desviante, exigindo grande esforço para resistir. Nas entrevistas realizadas nesta pesquisa, as idosas com diabetes relataram que sempre participavam no preparo de sua alimentação, compartilhando essa atividade com outros membros da família ou executando-a sozinha. Já para os idosos diabéticos, o preparo das refeições estava sob a responsabilidade de mulheres da família (esposa/filha/sobrinha).

Chama atenção que nas falas não foi identificado nenhum registro de consumo de alimentos diet e light. O que não implica, necessariamente, que não sejam consumidos. Mas a falta de espontaneidade em referi-los supõe que não fazem parte do cotidiano, seja por questões econômicas, de preferência individual, seja por questão cultural. Entende-se por diet aquele produto que está isento de um dos seus componentes, podendo ser o alimento sem açúcar, ou alternativamente ou concomitantemente, sem gordura, sem sal ou sem proteína. A legislação brasileira considera alimento light aquele que, em relação ao seu valor calórico, possui, no mínimo, $25 \%$ menos calorias do que o produto similiar convencional(25). Estudo realizado no Brasil identificou que os produtos diet e light são consumidos pelas famílias de classe alta, uma vez que os consumidores de baixa renda encontram barreiras para consumi-los devido ao seu alto custo em relação aos produtos convencionais ${ }^{(25)}$.

A ideia compartilhada pelos idosos entrevistados foi a de que é muito difícil seguir a dieta, que impõe uma ruptura cultural com as preferências relacionadas à alimentos como farinha de mandioca, carne de sol, feijoada, macaxeira, doces artesanais preparados com frutas regionais, dentre outros recordados com nostalgia e que são alimentos culturalmente nordestinos. Salienta-se que, mesmo diante das orientações dos profissionais sobre a dieta no tratamento do DM2, a decisão sempre é do usuário, competindo àqueles promover o empoderamento e a autonomia para que a pessoa com DM2 possa fazer melhores escolhas em relação à sua alimentação e, consequentemente, lograr melhor qualidade aos anos vividos.

Esta pesquisa teve como limitação o envolvimento de apenas um grupo de idosos com diabetes e que frequentava um único centro de saúde de um município do interior da Bahia. Apesar das limitações pontuadas, acredita-se que este estudo representa um avanço ao descrever às percepções dos idosos com DM2 quanto ao significado e práticas dietéticas. A vocalização sobre as percepções contribuiu para o alcance de princípios definidos pela PNPS ${ }^{(11)}$, como 0 de participação e autonomia, o que poderá favorecer a criação de espaços sociais que reflitam sobre o referido tema.

Este estudo poderá, ademais, nortear o planejamento de ações de promoção à saúde, bem como práticas de cuidados mais efetivas no gerenciamento do DM2 na pessoa idosa, não apenas no referido município, mas em outros locais do país, o que poderá permitir fortalecer especialmente a promoção de hábitos saudáveis de alimentação( ${ }^{(11)}$.

Entretanto recomenda-se que novos estudos sejam realizados abrangendo um grupo mais heterogêneo de participantes, a fim de obter uma visão ampliada sobre o tema em questão, como também pesquisar a efetividade de programas educativos inovadores nesse campo, fomentando a importância do desenvolvimento de diálogos entre gestores, trabalhadores e comunidade, para que possam refletir sobre essas morbidades da população com 
vista à composição de redes de compromisso e corresponsabilidade quanto à promoção da qualidade de vida da população e no cuidado com a saúde.

\section{CONSIDERAÇÕES FINAIS}

Este estudo possibilitou compreender os significados e as práticas dietéticas entre pessoas idosas com diabetes. Apesar da compreensão da importância da dieta para o tratamento do DM2, as decisões dos idosos diante das recomendações dietéticas são flutuantes, variadas e singulares. No que se refere aos significados atribuídos à dieta prescrita, observou-se que estão relacionados a sentimentos negativos e suas práticas demonstraram flexibilidade.

Os idosos com DM2 revelaram, ainda, facilidades e dificuldades no gerenciamento da dieta, notando-se que o manejo é central e complexo, assim como em outros grupos etários.

Os idosos pesquisados demonstraram que valorizam o prazer sensorial, ou seja, para eles é muito importante que a comida seja gostosa, atraente e que favoreça a sociabilidade. Ser idoso e ter DM2 implicou na necessidade de romper hábitos alimentares adotados no curso da vida, processo no qual a alimentação passou de uma prática prazerosa a angustiante, por isso encarada com tristeza e como mais uma perda vivenciada na velhice.

Recomenda-se que os profissionais de saúde envolvidos no cuidado à pessoa com DM2 e sua família cuidadora recebam formação e educação permanente, que contemplem a especificidade e a integralidade humana, e que as ações desenvolvidas sejam guiadas pela intra e intersetorialidade, interdisciplinaridade e inovação. Sugere-se, ainda, explorar ações educativas de promoção da saúde, tanto no âmbito individual quanto coletivo, para que possam ser ouvidas e problematizadas as experiências vividas nos eventos sociais com vistas a ajudá-los a encontrar soluções mais efetivas para os problemas que os afligem nessas ocasiões.

\section{CONFLITOS DE INTERESSE}

Não há neste manuscrito nenhuma relação que possa implicar em potencial conflito de interesses.

\section{FONTES DE FINANCIAMENTO}

A fonte de financiamento do artigo se deu pela Fundação de Amparo à Pesquisa do Estado da Bahia.

\section{CONTRIBUIÇÕES}

Nathiele Brasileiro de Souza Rocha e Andréa dos Santos Souza contribuíram com a elaboração e delineamento do estudo; a aquisição, análise e interpretação dos dados; e a redação e/ou revisão do manuscrito. Caliana Beatriz de Aguiar Barbosa contribuiu com a elaboração e delineamento do estudo; e a aquisição, análise e interpretação dos dados. Edméia Campos Meira, Juliana da Silva Oliveira e Isleide Santana Cardoso Santos contribuíram com a aquisição, análise e interpretação dos dados; e a redação e/ou revisão do manuscrito. Laiza Carvalho Costa contribuiu com a redação e/ou revisão do manuscrito.

\section{REFERÊNCIAS}

1. Oliveira JEP, Montenegro RM Jr., Vencio S, organizadores. Diretrizes da Sociedade Brasileira de Diabetes: 2017-2018. São Paulo: Ed Clannad; 2017.

2. International Diabetes Federation. IDF Diabetes Atlas [Internet]. 7th ed. Brussels, Belgium: International Diabetes Federation; 2017 [acesso em 2019 Out 03]. Disponível em: https://idf.org/e-library/epidemiologyresearch/diabetes-atlas.htm

3. Barsaglini RA, Canesqui AM. A alimentação e a dieta alimentar no gerenciamento da condição crônica do diabetes. Saúde Soc São Paulo. 2010;19(4):919-32.

4. Buchmann $M$, Wermeling M, Lucius-Hoene G, Himmel W. Experiences of food abstinence in patients with type2 diabetes: a qualitative study. BMJ Health Care Inform. 2016;6(1):e008907.

5. Habte BM, Kebede T, Fenta TG, Boon H. Explanatory models of adult patients with type 2 diabetes mellitus from urban centers of central Ethiopia. BMC Res Notes. 2016;9(1):441. 
6. World Health Organization. Global health risks: mortality and burden of disease attributable to selected major risks. Geneva: WHO; 2009.

7. Ministério da Saúde (BR), Secretaria de Vigilância à Saúde, Secretaria de Atenção à Saúde. Política Nacional de Promoção da Saúde: PNPS: revisão da Portaria MS/GM nº 687, de 30 de março de 2006. Brasília: Ministério da Saúde; 2015.

8. Minayo MCS. O desafio do conhecimento: pesquisa qualitativa em saúde. $12^{\mathrm{a}}$ ed. Rio de Janeiro: Hucitec; 2010.

9. Ministério da Saúde (BR), Secretaria de Atenção à Saúde, Departamento de Atenção Básica. Hipertensão Arterial Sistemica (HAS) e Diabetes Mellitus (DM): protocolo. Brasília: Ministério da Saúde; 2001.

10. Melo DM, Barbosa AJG. O uso do Mini-Exame do Estado Mental em pesquisas com idosos no Brasil: uma revisão sistemática. Cienc Saúde Colet. 2015;20(12):3865-76.

11. Ministério da Saúde (BR). Estratégias para o cuidado da pessoa com doença crônica. Brasília: Ministério da Saúde; 2014.

12. Nascimento LCN, Souza TV, Oliveira ICS, Moraes JRMM, Aguiar RCB, Silva LF. Theoretical saturation in qualitative research: an experience report in interview with schoolchildren. Rev Bras Enferm [Internet]. 2018 [acesso em 2019 Out 03];71(1):228-33. Disponível em: http://www.scielo.br/pdf/reben/v71n1/0034-7167reben-71-01-0228.pdf

13. Bardin L. Análise de conteúdo. Lisboa: Edições 70; 2011.

14. Castro-Sánchez AE, Ávila-Ortíz MN. Changing Dietary Habits in Persons Living With Type 2. J Nutr Educ Behav. 2013;45(6):761-66.

15. El-Sayed EF, Awadalla H, Noor SK, Elmadhoun WM, Sulaiman AA, Almobarak A O, et al. Sugar intake in Sudanese individuals was associated with some features of the metabolic syndrome: Population based study. Diabetes Metab Syndr. 2018;12(3):245-50.

16. García-Reza C, Campuzano EC, Torres DG, Tlachino MJGT, Garcia BCS. La percepción de un grupo de hombres sobre La Diabetes Mellitus: contribuciones a la enfermeira. Esc Anna Nery Rev Enferm. 2014;18(4):562-69.

17. Silva VP, Cardenas CJ. A comida e a sociabilidade na velhice. Rev Kairós. 2007;10(1):51-69.

18. Valentim SA, Haddad MCL, Rossaneis MA. Difficulties experienced by diabetesmellitus carriers residents in rural district. J Nurs UFPE. 2015;9(4):7330-7.

19. Dolina JV, BellatoR, Araújo LFS. O adoecer e morrer de mulher jovem com câncer de mama. Cienc Saúde Colet. 2013;18(9):2671-80.

20. Barbosa MAG, Almeida AMR, Figueiredo MA, Negromonte AG, Silva JSL, Viana MGS, et al. Alimentação e Diabetes mellitus: percepção e consumo alimentar de idosos no interior de Pernambuco. Rev Bras Promoç Saúde. 2015;28(3):370-78.

21. Silocchi $C$, Junges JR. Equipes de atenção primária: dificuldades no cuidado de pessoas com doenças crônicas não transmissíveis. Trab Educ Saúde. 2017;15(2):599-615.

22. Santos LM, Sampaio JRF, Borba VFC, Luz DCRP, Rocha BEM. Avaliação do hábito alimentar e estado nutricional de idosos com diabetes mellitus tipo 2 atendidos na atenção básica de saúde do município de Porteiras-CE. Rev E-Ciência. 2017;5(1):69-7.

23. Rodrigues HAF. Alimentação como fonte de sociabilidade e de hospitalidade. Rev Eletrônica Cienc Sociais. 2012;12(1):85-100.

24. Sawyer MT, Deines CK. Missingthe Mark With Latina Women With Type 2 Diabetes: Implications for Educators. Diabetes Educ. 2013;39(5):671-8.

25. Oliveira FCR, Hoffmann R. Consumo de alimentos orgânicos e de produtos light ou diet no Brasil: fatores condicionantes e elasticidades-renda. Segur Aliment Nutr. 2015;22(1):541-57. 
Endereço do primeiro autor:

Nathielle Brasileiro de Souza Rocha

Universidade Estadual do Sudoeste da Bahia - UESB

Av. José Moreira Sobrinho, s/n

Bairro: Jequiezinho

CEP: 45206-190 - Jequié - BA - Brasil

E-mail: nathi.brasileiro2@hotmail.com

\section{Endereço para correspondência:}

Andréa dos Santos Souza

Universidade Estadual do Sudoeste da Bahia - UESB

Av. José Moreira Sobrinho, $\mathrm{s} / \mathrm{n}$

Bairro: Jequiezinho

CEP: 45206-190 - Jequié - BA - Brasil

E- mail: andreassouza75@gmail.com

Como citar: Rocha NBS, Souza AS, Barbosa CBA, Meira EC, Oliveira JS, Santos ISC, et al. Significados e práticas dietéticas entre idosos diagnosticados com diabetes mellitus tipo 2. Rev Bras Promoç Saúde. 2019;32:9518. 